\title{
Systematic coarse graining: "Four lessons and a caveat" from nonequilibrium statistical mechanics
}

\author{
Hans Christian Öttinger* \\ ETH Zürich, Department of Materials, Polymer Physics, \\ HCI H 543, CH-8093 Zürich, Switzerland
}

\begin{abstract}
With the guidance offered by nonequilibrium statistical thermodynamics, simulation techniques are elevated from brute-force computer experiments to systematic tools for extracting complete, redundancy-free and consistent coarse grained information for dynamic systems. We sketch the role and potential of Monte Carlo, molecular dynamics and Brownian dynamics simulations in the thermodynamic approach to coarse graining. A melt of entangled linear polyethylene molecules serves us as an illustrative example.
\end{abstract}

*hco@mat.ethz.ch; http://www.polyphys.mat.ethz.ch/ 


\section{Goals}

The goal of coarse graining is to minimize the efforts required to solve a given problem. It moreover puts the focus on the essence of the problem and hence maximizes the depth of understanding. As coarse graining is the key to both efficiency and understanding, reliable general principles and procedures need to be established. We here summarize and illustrate a systematic approach based on nonequilibrium statistical mechanics. This theme article provides some useful guidelines for physically meaningful coarse graining. The author hopes to be thought-provoking, which may be more realistic for young scientists but equally important for experienced colleagues who have already carried out nonequilibrium simulations.

\section{Beyond Equilibrium Thermodynamics}

At equilibrium, statistical thermodynamics provides universally accepted recipes for coarse graining: (i) Thermodynamic potentials contain all the relevant information about thermodynamic systems in a compact format, and (ii) thermodynamic potentials can be calculated via partition functions. The ultimate task of computer simulations is to evaluate partition functions to obtain complete and consistent thermodynamic information. Simulations should not be regarded as computer experiments in which certain equations of state are "measured;" otherwise, one is at risk to violate the Maxwell relations between different equations of state instead of benefiting from the simplifications offered by these thermodynamic relationships.

Beyond equilibrium, we need to identify the generalizations of thermodynamic potentials as the primary sources of complete thermodynamic information. In response to this need, GENERIC ("general equation for the nonequilibrium reversible-irreversible coupling") offers a complete, coherent, and consistent theoretical framework of beyond-equilibrium thermodynamics. It is important to consider the energy $E$ and the entropy $S$ separately because they are the distinct generators of reversible and irreversible dynamics, respectively. In addition to the two generators $E$ and $S$, one needs a Poisson matrix $L$ that turns energy gradients into reversible dynamics and a friction matrix $M$ that turns entropy gradients into irreversible dynamics. The fundamental equation for the time evolution of the nonequilibrium variables 
$x$ is

$$
\frac{d x}{d t}=L(x) \cdot \frac{\delta E(x)}{\delta x}+M(x) \cdot \frac{\delta S(x)}{\delta x} .
$$

This equation is known as GENERIC [1, 2, 3]. It comes with a number of structural properties of the generators $E, S$ and the matrices $L, M$. For example, $L$ is always antisymmetric and $M$ is usually symmetric (more precisely, $M$ actually expresses the famous OnsagerCasimir symmetry of linear irreversible thermodynamics [3, 4] ). A strict separation of the reversible and irreversible contributions is provided by the mutual degeneracy requirements

$$
M(x) \cdot \frac{\delta E(x)}{\delta x}=0, \quad L(x) \cdot \frac{\delta S(x)}{\delta x}=0
$$

which are strong formulations of the conservation of energy even in the presence of dissipation and the conservation of entropy for any reversible dynamics. Entropy can only be produced by irreversible dynamics. A strong nonequilibrium generalization of the second law of thermodynamics is obtained by postulating that $M$ be positive-semidefinite.

Because $x$ typically contains position-dependent fields, such as the local mass, momentum and energy densities of hydrodynamics, the state variables are usually labeled by continuous (position) labels in addition to discrete ones. It is important to realize that the choice of the variables $x$, that is, the definition of an appropriate thermodynamic system for a given problem, is a step of crucial importance. Beyond equilibrium, and contrary to our equilibrium experience, this choice is far from obvious. Actually, this is the point at which the most physical intuition is required. A poor choice of $x$ cannot be repaired by even the most ingenious formulation of thermodynamic building blocks. For example, for the famous reptation model of a melt of entangled linear polymer molecules considered below, the idea

of smooth primitive paths and the corresponding configurational distribution function are the key to success.

\section{Beyond Equilibrium Statistical Mechanics}

The essence of nonequilibrium statistical mechanics and coarse graining is contained in Figure 1 for the entropy $S$ and Figure 2 for the friction matrix $M$. The entropy on the coarser level is obtained by counting the increasing number of microstates lumped into coarser states

for progressive coarse graining, and the friction matrix is associated with the increasing number of processes treated as fluctuations upon restricting the coarser description to slower 
and slower variables (according to the fluctuation-dissipation theorem, which holds even far beyond equilibrium, fluctuations are intimately related to dissipation or friction).

To classify and count microstates in the spirit of Figure 1, it is crucial to have a mapping $\Pi(z)$ that assigns a coarse grained state to any microstate $z$. In classical mechanics, the microstates $z$ are given by the positions and momenta of all particles. In a generalized microcanonical ensemble, all microstates $z$ associated with the same coarse configuration $\Pi(z)$ occur with the same probability so that the mapping $\Pi(z)$ fully characterizes the probability density $\rho_{x}(z)$ to find a microstate $z$ for given coarser variables $x$. In any ensemble, these variables $x$ are the averages of $\Pi(z)$ evaluated with the probability density $\rho_{x}(z)$, i.e. $x=\langle\Pi(z)\rangle_{x}$.

Like at equilibrium, it is convenient to pass from microcanonical to canonical ensembles to simplify practical calculations. One then controls the averages of $\Pi(z)$ to be $x$ (or some of them) by a corresponding set of Lagrange multipliers $\lambda$. The passage from one ensemble to another one works exactly as in equilibrium statistical mechanics, and it is closely related to Legendre transformations. The only difference is that the nonequilibrium list of variables is usually much longer and less universal than the list of equilibrium variables. For a larger list of variables, the number of microstates per coarse grained state goes down, so that the statistical equivalence of different ensembles needs to be investigated more carefully than at equilibrium.

The concrete statistical recipe behind Figure 1 is that the entropy of a coarse state is given by the logarithm of the number of associated microstates. Figure 1 visualizes the pattern recognition process behind lumping microstates into coarser states in a simplified cartoon. In general, a suitable representation needs to be identified before any clustering of microstates can be recognized, which is again the art of finding suitable variables. Figure 2 illustrates the essence of the friction matrix and actually represents the formula

$$
M(x)=\frac{1}{k_{\mathrm{B}}} \int_{0}^{\tau}\left\langle\dot{\Pi}^{\mathrm{f}}(z(t)) \dot{\Pi}^{\mathrm{f}}(z(0))\right\rangle_{x} d t,
$$

where $k_{\mathrm{B}}$ is Boltzmann's constant, $\tau$ is an intermediate time scale separating the slow degrees of freedom from the fast degrees of freedom, $\dot{\Pi}^{\mathrm{f}}(z)$ is the rapidly fluctuating part of the rate of change of the atomistic expressions $\Pi(z)$ for the slow variables $x$, and the average is over an ensemble of atomistic trajectories $z(t)$ consistent with the coarse grained state $x$ at $t=0$ and evolved according to the fast contribution to the atomistic dynamics to the time $t$. 
Equation (3) is known as a Green-Kubo formula [5, 6].

The GENERIC building blocks $E$ and $L$ associated with reversible dynamics are obtained by straightforward averaging of the microscopic energy and of the Poisson bracket of classical mechanics for the components of $\Pi(z)$. We thus have a complete set of recipes to calculate the four GENERIC building blocks of nonequilibrium thermodynamics from statistical mechanics. All the building blocks and their properties have been derived from Hamilton's equations of motion by separating the slow and fast degrees of freedom with the projection-operator method [3, 6, 7]. As already suggested by Figures 1 and 2, the statistical recipes can be generalized to progressive coarse graining rules so that it is not necessary to start always from the atomistic level. Time occurs only in the expression (3) for the friction matrix $M$; all other building blocks are obtained by time-independent averaging or counting according to the statistical ensemble. All dynamic material information must hence be contained in $M$.

We conclude this section on nonequilibrium statistical mechanics with a warning that is obvious within the current approach (remember Figures 1 and 2), but is often ignored in the literature on coarse graining:

Caveat: The hallmark of true and complete coarse graining is an increase of both entropy and dissipation (or friction); meaningful coarse graining techniques focus on calculating both increases or, at least, are capable of accounting for the corresponding effects.

The passage from reversible to irreversible equations is one of the big intellectual achievements associated with Boltzmann's kinetic equation for rarefied gases. Such a transition from reversible to irreversible (or, the emergence of a nonzero friction matrix) must occur in any coarse graining starting from the reversible atomistic level. Even when starting from a level with an irreversible contribution to the dynamics, additional irreversibility must be born in a coarse graining process (otherwise, we have a solution technique without any elimination of fast processes in favor of fluctuations). The issue of coarse graining versus reduction (i.e., solving) is a hot topic in mathematical physics [8].

In many cases, one tries to coarse grain systems by introducing temperature-dependent effective potentials (more precisely: free energies) for "superatoms" which typically consist of some 10 atoms. To calculate such effective potentials efficiently, "iterative Boltzmann inversion" has been developed. With this approach, one can then handle the additional 
entropy appropriately, but not the additional dissipation, the emergence of (further) irreversibility. In many cases, the effective potentials are simply used in the reversible equations of motion of classical mechanics to perform molecular dynamics simulations (see [9, 10] and references therein). A simplistic attempt to allow at least for some kind of dissipative effect would be to renormalize time [9, 11] or to introduce frictional forces into the equations of motion [12]. The example of hydrodynamic interactions in polymer solutions, however, warns us that the non-scalar nature of the friction matrix $M$ is crucial to find many of the relevant physical effects such as second-normal stress differences in shear flow [13, 14]. The entire wealth of dynamic phenomena is actually contained in $M$, so that a simple rescaling of time or a scalar friction can only be of limited value.

\section{The Lessons (for Beginners and Professionals)}

It is worthwhile to emphasize once more that identifying the proper relevant variables is the key to success in any systematic coarse graining method. The initial step is hence clear:

Lesson 1: Identify a suitable set of relevant variables for a coarser target level and express them in terms of the variables of a finer source level.

The finer level is considered to be well-established. In many cases, it is the atomistic level with particle positions and momenta as variables. In some cases, the art of finding appropriate force fields is supported by quantum calculations.

The implications of "Lesson 1" are dramatic: We cannot start a simulation before we have found suitable coarse grained variables, that is, before we have reached a basic understanding of our problem of interest. This is the price to pay for the possibility to do systematic and iterated coarse graining to bridge a wide range of scales. In brute-force computer experiments, the system is driven by boundary conditions, nonequilibrium initial conditions, and/or external forces chosen to mimic the experimental situation of interest. One then "measures" some information of direct interest. In statistical mechanics, the system is considered in terms of well-defined nonequilibrium ensembles and they involve the coarse grained variables explicitly.

In the presence of self-similarity or universality (or in the absence of better ideas), one takes a target level of the same type as the source level. This is, for example, the idea 
behind "superatoms" (without any justification by self-similarity or universality). In general, ingenious ideas about suitable target levels promise a more rewarding starting point for significant coarse graining. A variety of inspiring ideas has been collected in a recent monograph by Kröger [15].

The choice of variables determines the observables to be averaged to obtain the thermodynamic building blocks in which the complete coarse grained information is collected. Moreover, the form of the statistical ensemble depends strongly on the chosen variables.

Lesson 2: Choose a convenient nonequilibrium ensemble, verify its equivalence with a generalized microcanonical ensemble, interpret all the Lagrange multipliers, and find their values for nonequilibrium situations of interest.

In general, the identification of the proper values of the Lagrange multipliers requires an iterative procedure. For example, the Lagrange multipliers required to describe the structure of a complex fluid undergoing homogeneous steady flow can only be related to the velocity gradients if the friction matrix $M$ is known [16] (from an iterative procedure leading to self-consistency). The highly advantageous restriction to homogeneous situations is often possible due to the local character of the thermodynamic building blocks. Biased Monte Carlo methods, parallel tempering [17, 18], multicanonical methods [19] and densityof-states Monte Carlo [20, 21, 22] offer interesting possibilities for locating self-consistent values of Lagrange multipliers efficiently.

Lesson 3: Calculate the static building blocks $E, S$, and $L$ of nonequilibrium thermody-
namics by the highly developed Monte Carlo methods of equilibrium statistical mechanics
$[23,24,25]$. Overcome the challenges implied by a wide range of scales in a problem by a
balanced set of Monte Carlo moves causing configurational changes occurring on various
length and time scales.

In more provocative words, the most unphysical Monte Carlo moves, such as breaking and recombining polymer chains, are most promising for overall computational efficiency. Whenever Monte Carlo time can be assumed to be proportional to real time, this is a guarantee for computational inefficiency.

In many cases, the Poisson matrix $L$ can be obtained analytically from the transformation behavior of the variables under space transformations without performing any simulations. 
Then, only energy and entropy need to be found from Monte Carlo simulations.

Lesson 4: Evaluate the friction matrix $M$ from the Green-Kubo formula (3). As the only building block containing dynamic material information, its calculation requires dynamic simulation techniques. However, dynamic simulations are required only for a small fraction of the longest time scales of the system. The only goal of long-time stabilization is to conserve the chosen statistical ensemble.

If one starts from the atomistic level, molecular dynamics simulations [26] are the natural tool. Starting from a coarse grained level, for which fast processes are treated as white noise, the equations of motion become stochastic differential equations and Brownian dynamics simulations are required [27].

It is often stated that the reliable determination of dynamic material properties such as the diffusion coefficient requires trajectories that are large compared to the longest relaxation time of the system. Statistical mechanics tells us that all the relevant information is actually obtained within a fraction of a relaxation time. The Green-Kubo formula (3) for the friction matrix, which is the only thermodynamic building block containing dynamic material information, uses the intermediate time scale $\tau$ as the upper limit of integration. This time scale is long from the perspective of the fast processes treated as fluctuations (correlations of fluctuations decay to zero within $\tau$ ) and short from the perspective of the relevant slow processes (they are arrested). Typically, the longest relaxation time and the intermediate time scale $\tau$ are not known in advance. We hence try to estimate the friction matrix from increasingly longer trajectories until stable results are obtained, which typically determine the characteristic relaxation times of a system. We can then check whether the required length of the trajectory is much shorter than the longest relaxation times so that there actually exists a separating time scale $\tau$ inbetween.

Taking "Lesson 4" to heart typically is rewarded with two orders of magnitude in computational efficiency. This miracle happens because we do not need to reveal the relevant relaxation mechanisms in thermodynamically guided simulations; they are implied by the choice of variables, emphasizing once more how important this initial choice ("Lesson 1") is. To check the adequacy of the choice of variables, we should employ the coarse grained level to make predictions for a variety of specific situations, which we can compare to simulations on the more detailed level or to experiments. 
In most dynamic simulations, one tries to produce very long trajectories. The practical advantage is that one then does not need any separate Monte Carlo simulations. From the viewpoint of efficiency, however, one loses the benefit of "Lesson 3" that Monte Carlo can beat the time scale problem. If one performs these long dynamic simulations to avoid Monte Carlo, one has to stabilize the long-time dynamics, for example by thermostats, to conserve the chosen statistical ensemble (see Section 8.4.3 of [3] and references therein). For that purpose, the ideas behind the Nosé-Hoover [28, 29] and Nosé-Poincaré [30, 31] thermostats as well as Andersen's barostat [32] are particularly useful. Any modification of the atomistic equations of motion is allowed as long as it has no significant influence on the dynamics on time scales shorter than $\tau$, that is, on the dynamic material information. Actually, also in the formal projection-operator results, the slow degrees of freedom are projected out of the time evolution in the Green-Kubo formula.

\section{Example: Entangled Polyethylene}

In the spirit of "Lesson 1", we need a suitable coarse grained structural variable before we can start a thermodynamically guided simulation technique. Our choice for entangled linear polymer chains is motivated by the famous reptation model of Doi and Edwards [33]. From the random coil conformations of the atomistic polymer chains we construct smooth primitive paths or worms (see Figure 3) which allows us to define a tangent unit vector at any position $\sigma$ along the chain $(0 \leq \sigma \leq 1)$ [34]. The probability density $f(u, \sigma)$ serves as our structural variable.

The corresponding Lagrange multiplier $\lambda(u, \sigma)$ in a generalized canonical ensemble ("Lesson 2") can be identified as an "effective potential" characterizing the deviation from the equilibrium probability density via $f=f_{\mathrm{eq}} e^{-\lambda}$, where $f_{\mathrm{eq}}$ is just a constant for the reptation model. The simplicity of this relationship is a consequence of the simple form of Boltzmann's entropy for probability densities. With the chosen structural variable, we fully rely on the information about the orientation $u$ of a single segment $\sigma$ without any correlation between different segments or different chains. Then, the stiffness of the worms enters as an external parameter equivalent to the number of entanglements per chain. To avoid that, one would need to elevate the number of entanglements to the level of a dynamic variable. After borrowing the ingenious structural variable $f(u, \sigma)$ from Doi and Edwards and choosing a 
convenient ensemble, the way to thermodynamically guided simulations is straightforward. No further intuition is required. More details on the example sketched in the following can be found in Section 8.4.6 of [3].

Figure 3 suggests naturally that, in addition to the contour of the worms, one might consider the worm cross section as a relevant variable. Such a modification of the DoiEdwards model has previously been suggested to explain the experimental results for the second normal-stress difference in shear flow [35, 36, 37].

Even before we start a simulation, a number of further conclusions can be drawn from closer inspection of the statistical expressions for the thermodynamic building blocks. There is no energy associated with the variable $f(u, \sigma)$, and the entropy associated with any probability density is expected to be of the Boltzmann type. Therefore, for the single-segment reptation model, the stresses in a polymer melt are entirely entropic in nature. Note that such a statement depends on the level of description. On the atomistic level, the very same stresses are obtained from the interactions between all atoms of all chains.

To construct the Poisson matrix, we only need to know that the orientation vector $u$ is rotated by the flow field. The only quantity to be determined from simulations is the friction matrix. As all static building blocks are known, there is no need for Monte Carlo simulations ("Lesson 3"). We here determine the friction matrix at equilibrium, that is, for $\lambda=0$, by means of a molecular dynamics simulation with a well-established force field for polyethylene [38, 39, 40] ("Lesson 4"). The structural variable then does not occur in the definition of the ensemble, but it still determines the correlation functions to be considered in the GreenKubo formula (3). Analytical rearrangements of the Green-Kubo formula show that we need to find second moments of displacements of $\sigma$ and $u$ up to intermediate time scales, short compared to the longest relaxation time which is also known as the disengagement or reptation time.

Orientational diffusion of $u$ has actually been proposed as a "constraint-release mechanism" [41] that is absent from the original Doi-Edwards model. We here focus on the diffusion of $\sigma$, which is the diffusion of worms along their contours and thus the classical reptation mechanism. The fact that we need to focus on the reptative motion of the worms is a straightforward result of the thermodynamically guided approach carried out for the structural variable $f(u, \sigma)$. There is no need to look at any other dynamic quantity, such as the stress tensor or center-of-mass diffusion, unless one wants to investigate the adequacy 
of the coarse grained level.

Figure 4, which is based on data kindly provided by Vlasis Mavrantzas, shows the average square displacement of $\sigma$ as a function of time for polyethylene chains with 1000 carbon atoms per chain. The slope in this figure determines the frictional properties and hence the disengagement time, which is obtained as $(6.8 \pm 0.2) \mu \mathrm{s}$. Note that this result is obtained from a trajectory of less than $0.5 \mu \mathrm{s}$. This is a striking example of "Lesson 4": The frictional properties on the coarse grained level, which determine the disengagement time, can be obtained reliably on a much shorter, intermediate time scale. Total disengagement requires $\left(\Delta_{t} \sigma\right)^{2}$ to be of order unity; it is hence clear from Figure 4 that the disengagement time is indeed determined from the early traces of reptation becoming visible through the apt choice of smooth primitive paths as variables. To obtain the same material information from the center-of-mass diffusion, one needs trajectories comprising several relaxation times. This gain of efficiency hopefully is a convincing example for the benefits of learning the lessons of nonequilibrium statistical mechanics. Equally important is the gain of understanding provided by the reptation model of Doi and Edwards, which enables the computational efficiency of systematic coarse graining.

In conclusion, the best strategy for thermodynamically guided, systematic coarse graining requires a combination of Monte Carlo simulations for the efficient calculation of energy and entropy and dynamic simulations for the friction matrix. Monte Carlo simulations can also provide the initial conditions for dynamic simulations. Stand-alone Monte Carlo simulations are usually of limited value for nonequilibrium systems because the identification of the Lagrange multipliers describing deviations from equilibrium requires dynamic material information [16]. In spite of the rapidly developing computer technology and the increasing temptation to solve all engineering problems by brute-force computer experiments, the deeper understanding required and enriched by systematic coarse graining will hopefully be appreciated in perpetuity.

\section{Acknowledgments}

I thank Juan José de Pablo for inviting and inspiring me to write this theme article. My long-standing, enjoyable and fruitful collaboration with Vlasis Mavrantzas and Martin Kröger contributed enormously to the development of systematic coarse graining procedures. 
I greatly appreciate Dieter Schlüter's constructive comments which helped to intensify my messages.

[1] M. Grmela and H. C. Öttinger, Phys. Rev. E 56, 6620 (1997).

[2] H. C. Öttinger and M. Grmela, Phys. Rev. E 56, 6633 (1997).

[3] H. C. Öttinger, Beyond Equilibrium Thermodynamics (Wiley, Hoboken, 2005).

[4] S. R. de Groot and P. Mazur, Non-Equilibrium Thermodynamics, 2nd ed. (Dover, New York, 1984).

[5] R. Kubo, M. Toda, and N. Hashitsume, Nonequilibrium Statistical Mechanics, Vol. II of Statistical Physics, 2nd ed. (Springer, Berlin, 1991).

[6] H. Grabert, Projection Operator Techniques in Nonequilibrium Statistical Mechanics (Springer, Berlin, 1982).

[7] H. C. Öttinger, Phys. Rev. E 57, 1416 (1998).

[8] Model Reduction and Coarse-Graining Approaches for Multiscale Phenomena, edited by A. N. Gorban, N. Kazantzis, I. G. Kevrekidis, H. C. Öttinger, and C. Theodoropoulos (Springer, Berlin, 2006).

[9] G. Milano and F. Müller-Plathe, J. Phys. Chem. B 109, 18609 (2005).

[10] Q. Sun and R. Faller, Macromolecules 39, 812 (2006).

[11] V. A. Harmandaris, N. P. Adhikari, N. F. A. van der Vegt, and K. Kremer, Macromolecules 39, 6708 (2006).

[12] J. T. Padding and W. J. Briels, J. Chem. Phys. 117, 925 (2002).

[13] H. C. Öttinger, J. Chem. Phys. 86, 3731 (1987).

[14] H. C. Öttinger, J. Chem. Phys. 90, 463 (1989).

[15] M. Kröger, Models for Polymeric and Anisotropic Liquids, No. 675 in Lecture Notes in Physics (Springer, Berlin, 2005).

[16] V. G. Mavrantzas and H. C. Öttinger, Macromolecules 35, 960 (2002).

[17] E. Marinari and G. Parisi, Europhys. Lett. 19, 451 (1992).

[18] M. C. Tesi, E. J. Janse van Rensburg, E. Orlandini, and S. G. Whittington, J. Stat. Phys. 82, 155 (1996).

[19] R. Faller, Q. Yan, and J. J. de Pablo, J. Chem. Phys. 116, 5419 (2002). 
[20] F. Wang and D. P. Landau, Phys. Rev. Lett. 86, 2050 (2001).

[21] F. Wang and D. P. Landau, Phys. Rev. E 64, 056101, 1 (2001).

[22] R. Faller and J. J. de Pablo, J. Chem. Phys. 119, 4405 (2003).

[23] D. P. Landau and K. Binder, A Guide to Monte Carlo Simulations in Statistical Physics (Cambridge University Press, Cambridge, 2000).

[24] K. Binder and D. W. Heermann, Monte Carlo Simulation in Statistical Physics, Springer Series in Solid-State Sciences, Volume 80, 2nd ed. (Springer, Berlin, 1992).

[25] O. G. Mouritsen, Computer Studies of Phase Transitions and Critical Phenomena, Springer Series in Computational Physics (Springer, Berlin, 1984).

[26] M. P. Allen and D. J. Tildesley, Computer Simulation of Liquids (Clarendon, Oxford, 1987).

[27] H. C. Öttinger, Stochastic Processes in Polymeric Fluids: Tools and Examples for Developing Simulation Algorithms (Springer, Berlin, 1996).

[28] S. Nosé, Mol. Phys. 52, 255 (1984).

[29] W. G. Hoover, Phys. Rev. A 31, 1695 (1995).

[30] S. D. Bond, B. J. Leimkuhler, and B. B. Laird, J. Comput. Phys. 151, 114 (1999).

[31] B. Leimkuhler, Comput. Phys. Commun. 148, 206 (2002).

[32] H. C. Andersen, J. Chem. Phys. 72, 2384 (1980).

[33] M. Doi and S. F. Edwards, The Theory of Polymer Dynamics, International Series of Monographs on Physics, Volume 73 (Clarendon, Oxford, 1986).

[34] M. Kröger, J. Ramírez, and H. C. Öttinger, Polymer 43, 477 (2002).

[35] G. Ianniruberto and G. Marrucci, J. Non-Newtonian Fluid Mech. 79, 225 (1998).

[36] G. Marrucci and G. Ianniruberto, J. Non-Newtonian Fluid Mech. 82, 275 (1999).

[37] H. C. Öttinger, J. Non-Newtonian Fluid Mech. 89, 165 (2000).

[38] V. A. Harmandaris, V. G. Mavrantzas, and D. N. Theodorou, Macromolecules 31, 7934 (1998).

[39] V. A. Harmandaris, V. G. Mavrantzas, and D. N. Theodorou, Macromolecules 33, 8062 (2000).

[40] V. A. Harmandaris, V. G. Mavrantzas, D. N. Theodorou, M. Kröger, J. Ramírez, H. C. Öttinger, and D. Vlassopoulos, Macromolecules 36, 1376 (2003).

[41] H. C. Öttinger, Phys. Rev. E 50, 4891 (1994). 


\section{Figures}

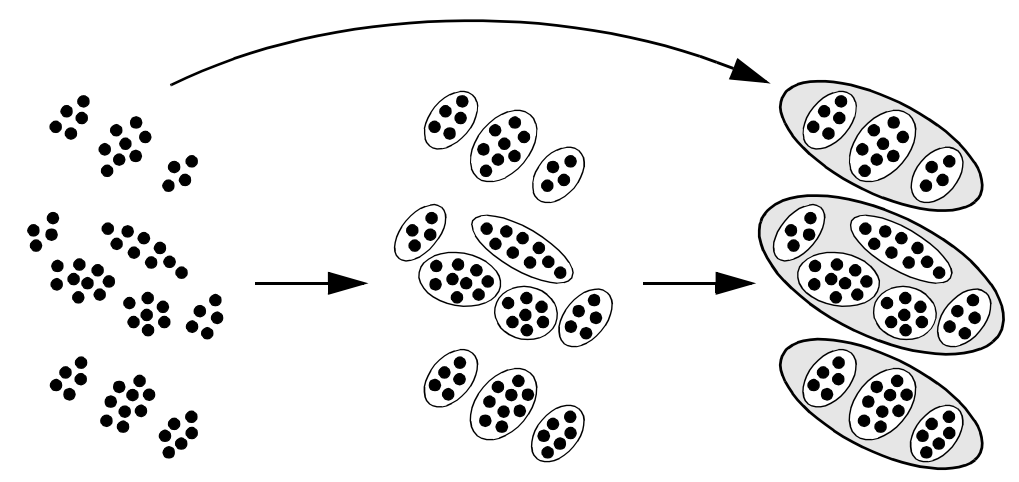

Figure 1. Grouping of microstates (black circles) into progressively coarser states (ellipses); following well-established equilibrium ideas, the nonequilibrium entropy of a coarse state is given by the logarithm of the number of microstates in the corresponding ellipse.

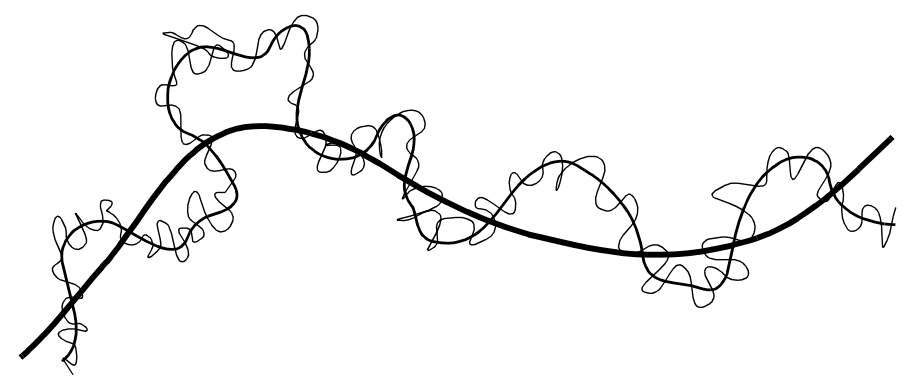

Figure 2. Trajectories on three levels of description; an increasing number of fast processes are eliminated in favor of random fluctuations (not shown in the figure) for the coarser descriptions (for increasing line thickness). 


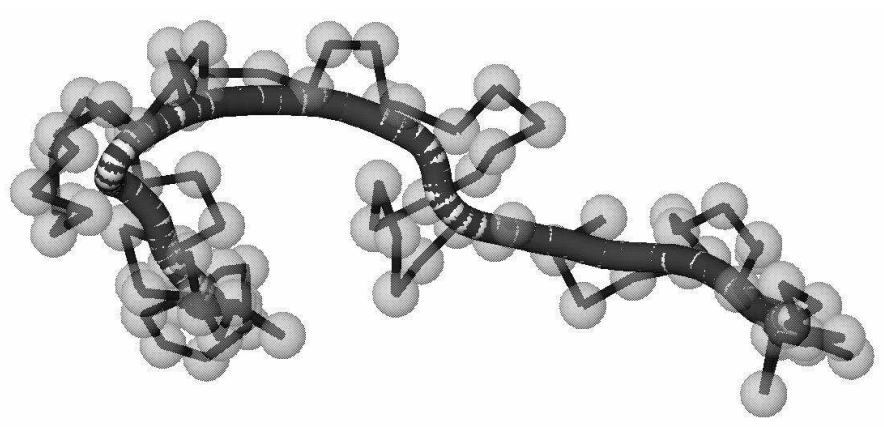

Figure 3. Competing-spring construction of a smooth primitive path from an atomistic chain; the smoothed worm possesses a local tangent vector. [Figure courtesy of M. Kröger, ETH Zürich.]

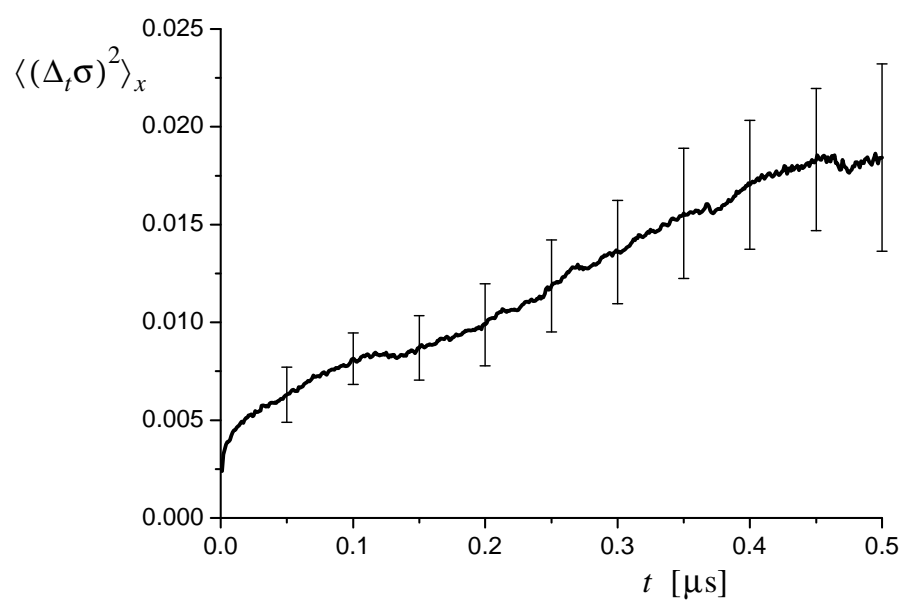

Figure 4. Diffusion along the primitive path constructed for a polyethylene chain with 1000 carbon atoms; the slope represents the dynamic material information for the reptation model. [Figure courtesy of M. Kröger, ETH Zürich.] 